\title{
Labor dystocia and oxytocin augmentation before or after six centimeters cervical dilatation, in nulliparous women with spontaneous labor, in relation to mode of delivery
}

Cecilia Brüggemann

Linköping University

Sara Carlhäll

Linköping University

Hanna Grundström

Linköping University

Marie Blomberg ( $\square$ marie.blomberg@liu.se )

Linköping University

\section{Research Article}

Keywords: oxytocin augmentation, active labor, labor dystocia, cesarean section, birth experience

Posted Date: February 10th, 2022

DOI: https://doi.org/10.21203/rs.3.rs-1280400/v1

License: (c) (i) This work is licensed under a Creative Commons Attribution 4.0 International License.

Read Full License 


\section{Abstract}

Background: The effects of diagnosing and treating labor dystocia with oxytocin-infusion at different cervical dilatations are not fully evaluated. Therefore, we aimed to examine whether cervical dilatation at diagnose of dystocia and initiation of oxytocin-infusion was associated with mode of birth, obstetric complications and women's birthing experience.

Method: A retrospective cohort study, performed in a low cesarean section setting, included 588 nulliparous term women with spontaneous onset of labor and dystocia requiring oxytocin augmentation. The study population was divided into three groups according to cervical dilatation at diagnose of dystocia and initiation of oxytocin-infusion ( $\leq 5 \mathrm{~cm}, 6-10 \mathrm{~cm}$, fully dilated) with mode of birth as the primary outcome. Secondary outcomes were obstetrical and neonatal complications and women's experience of childbirth. The risk of operative birth was assessed using binary logistic regression with suitable adjustments (maternal age, body mass index and risk assessment at admission to the labor ward). The $6-10 \mathrm{~cm}$ group was set as reference.

Results: Cesarean section was performed in $12 \%$ in the $\leq 5 \mathrm{~cm}$ group, $6 \%$ in the $6-10 \mathrm{~cm}$ group and $0 \%$ in the fully dilated group $(p<.001)$, with no differences in the instrumental birth rates $(11-14 \%, p=.569)$. There was no increased risk for operative birth (cesarean and vacuum) in the $\leq 5 \mathrm{~cm}$ group compared to the 6$10 \mathrm{~cm}$ group, adjusted OR 1.28 (0.78-2.08). The fully dilated group had a decreased risk of operative birth (adjusted OR $0.4895 \% \mathrm{Cl} 0.27-0.85$ ). The rate of a negative birthing experience was high in all groups (28.5\%, $19 \%$ and $18 \%$ ) and the risk was increased among women in the $\leq 5 \mathrm{~cm}$ group compared with the 6-10 cm group, adjusted OR 1.76 (95\% $\mathrm{Cl} 1.05-2.95)$.

Conclusions: Women with dystocia requiring oxytocin augmentation diagnosed in second stage of labor had an increased chance of spontaneous vaginal birth compared with women requiring oxytocin augmentation in the late first stage of labor. No differences were shown between early and late active phase of first stage of labor concerning operative birth. Although, women with dystocia requiring oxytocin augmentation at $\leq 5 \mathrm{~cm}$ cervical dilatation seemed to have an increased risk of a negative birth experience.

\section{Introduction}

Labor dystocia is a common complication in nulliparous women and strongly related to cesarean section (CS) and instrumental vaginal delivery (1-3). The absolute majority of women diagnosed with labor dystocia receive oxytocin infusion to enhance uterine contractions $(4,5)$. Currently, the definition of labor dystocia at different stages of labor is about to change. The American College of Obstetricians and Gynecologists (ACOG) $(6)$ and the World Health Organization (WHO) $(7,8)$ suggests that active labor start at 5 or $6 \mathrm{~cm}$ cervical dilatation, compared with the traditional definition by Friedman (9) stating 3-4 cm as the threshold for start of active labor. Besides that active labor seems to start at a higher cervical 
dilatation, the view of what is normal labor progress is debated and new cut offs for protracted labor at different cervical dilatations has been suggested (7).

The WHO and ACOG both changed their definitions of start of active labor (recommendations) to stem the rising CS rates, as too much focus on cervical progression in early labor was viewed as a risk factor for CS and other interventions in labor that may negatively affect maternal and neonatal outcomes $(6,7)$. Recommendations in Sweden still adhere to Friedman's' definition of active labor $(4 \mathrm{~cm}$ cervical dilatation and expected cervical dilatation rate of $1 \mathrm{~cm} /$ hour from $4 \mathrm{~cm}$ with some slight moderations) (10).

The CS rates in nulliparous women with a singleton term ( $\geq 37+0$ gestational weeks) pregnancy, spontaneous onset of labor and vertex presentation, e.g. the Ten Group Classification System (TGCS) Group $1(11,12)$ are relatively low in Sweden (range in 2020, 3-11\%) compared with many other countries (13). In Sweden, Region Östergötland has one of the lowest rates (5.9\% in 2020). In this region an active improvement project, focusing on increasing the rate of spontaneous vaginal births in TGCS group 1 women, has been performed (12-14).

It is still not clear whether diagnosing and treating labor dystocia with oxytocin before 5-6 cm cervical dilatation increases the risk of CS compared with after 5-6 cm (15), and an important question that arises is whether results from studies performed in a high CS rate context could be extrapolated to settings with low numbers of interventions during labor? A change of the definition of active labor and permission of a slower labor progress needs to be thoroughly evaluated in relation to mode of delivery and other outcomes in different contexts before the definition is fully implemented. In Region Östergötland, labor dystocia has been diagnosed and treated with oxytocin infusion based on the criteria of a cervical dilatation speed of $1 \mathrm{~cm} /$ hour, before as well as after $5-6 \mathrm{~cm}$ cervical dilatation, since long. Therefore, this setting was suitable for a comparison of different cervical dilatation groups concerning mode of delivery. Thus, the primary aim of this study was to evaluate the association between cervical dilatation at diagnosing labor dystocia and initiating oxytocin infusion for labor augmentation and mode of birth in nulliparous women at term with a singleton pregnancy, vertex presentation and spontaneous onset of labor. Secondary outcomes were obstetrical and neonatal outcomes and women's experience of childbirth.

\section{Method}

This retrospective cohort study included nulliparous women with a singleton and term $(\geq 37+0$ gestational weeks) pregnancy, spontaneous onset of labor and vertex presentation, TGCS Group 1 (12) who gave birth from March to November 2018 at two hospitals in the Region Östergötland, which has approximately 5000 births per year combined. Further inclusion criteria were a documented risk classification on admission to the labor ward, a diagnose of labor dystocia and initiation of oxytocin infusion in labor.

The participating hospitals followed the same national clinical guidelines concerning risk classification, active labor care, diagnose of labor dystocia and oxytocin infusion initiation. Maternal and fetal risk 
classification was performed on admission to the labor ward using three risk categories: low, medium or high risk (Appendix 1). Active labor was defined as $4 \mathrm{~cm}$ of cervical dilatation, or one $\mathrm{cm}$ of cervical dilatation and a completely effaced cervix, painful, regular contractions and/or rupture of the membranes and progress of cervical dilatation within the following two hours, in accordance with the Swedish national recommendations (10). Also in accordance with Swedish national guidelines, diagnose of labor dystocia and initiation of oxytocin infusion was indicated when there was a delay in the expected cervical progress of one $\mathrm{cm} /$ hour for more than three hours, no progress in descent of the fetal head for one hour when fully dilated, or no progress after pushing actively for 30 minutes (5).

Maternal, obstetric and neonatal data were prospectively recorded in standardized electronic medical records (Obstetrix ${ }^{\circledR}$ ). The maternal characteristics assessed were age, height and weight in early pregnancy, smoking during pregnancy, diabetes mellitus, hypertension, and asthma/lung disease. Maternal height and weight were measured at the first antenatal visit in gestational weeks 8-12, which enabled calculation of early pregnancy Body Mass Index $\left(\mathrm{BMI}, \mathrm{kg} / \mathrm{m}^{2}\right)$. The obstetric characteristics that were extracted were: gestational age at birth, active labor time estimates, cervical dilatation at the start of oxytocin augmentation, epidural analgesia, mode of birth, occurrence of obstetric anal sphincter injury (OASI), postpartum hemorrhage (PPH) and women's birthing experience by Visual analogue scale (VAS). At the postnatal ward, all women were asked to assess their overall birthing experience as a VAS-score ranging from 1 to 10 , where 1 is a very negative experience and 10 is a very positive experience. This assessment of childbirth experience by VAS is a well-established routine in the postnatal care at the participating delivery units included in this study. A value of VAS 1-4 was considered a negative birthing experience according to the Swedish Pregnancy Registry (16).

Furthermore, risk classification on admission (low, medium, high) was also extracted (Appendix 1). The risk classification partly assesses the risk of labor dystocia by including parameters such as first trimester $\mathrm{BMI} \geq 30$, prolonged latent phase of labor, maternal psychological well-being, hypertensive disorders and preeclampsia, and fetal well-being (i.e., risk for infections in the newborn, intra uterine growth retardation, non-reassuring CTG (Cardiotocography) and heavily meconium-stained waters). The neonatal variables assessed were fetal birth weight and Apgar score $<7$ at five minutes and umbilical cord arterial $\mathrm{pH}<7.10$.

The study population was categorized according to cervical dilatation at diagnose of labor dystocia and start of oxytocin infusion as follows: $\leq 5 \mathrm{~cm}, 6-10 \mathrm{~cm}$ and fully dilated.

The primary outcome was mode of birth (spontaneous vaginal birth, instrumental vaginal birth, or CS) and a composite outcome of operative birth including both CS and instrumental birth. Secondary outcomes were the use of epidural anesthesia, OASI grades III and IV, PPH $>1,000 \mathrm{~mL}$, negative childbirth experience defined as VAS 1-4 and Apgar score $<7$ at five minutes and cord arterial $\mathrm{pH}<7.10$.

The sample size calculation using Fischer's exact test was based on rates of operative birth in TGCS group 1 in 2017 at the hospitals. With 183 women in each group (cervical dilatation $\leq 5 \mathrm{~cm}$ and 6-10 cm) 
a difference in rate of operative births ( 8 vs $17 \%$ ) could be detected with a 0.05 level of significance at a power of $80 \%$

The study was approved by the Regional Ethical Review Board in Linköping, Sweden on June $14^{\text {th }}, 2017$ (Dnr 2017-277-31) and on February 19th, 2019 (Dnr: 2019-01059).

\section{Statistical analyses}

All analyses were performed using SPSS Statistical package version 25.0 (IBM Corporation 1989, 2017). Maternal characteristics and obstetric and neonatal outcomes were analyzed using a $\mathrm{Chi}^{2}$ test for categorical variables and when appropriate Fischer's exact test, and a one-way ANOVA (Analysis of Variance) for continuous variables. A p-value of $<0.05$ was considered statistically significant. Binary logistic regression was performed to calculate odds ratios (ORs), adjusted odds ratios (aORs) and 95\% confidence intervals $(95 \% \mathrm{Cls})$ for primary and secondary outcomes. The reference group was set at 6-10 $\mathrm{cm}$ according to the definition of active labor by ACOG (6). In the binary logistic regression, CS and instrumental vaginal birth were merged into the outcome operative birth due to the fact that there was no $\mathrm{CS}$ in the fully dilated group. The results were adjusted for maternal age at birth, BMI in early pregnancy and risk classification on admission to the labor ward.

\section{Results}

A total number of 588 women were included in the study. The women eligible for the study and the women who were excluded are presented in Figure 1. A total number of $242(41 \%)$ women were classified as low risk at admission to the labor ward and $346(59 \%)$ as medium risk. None of the women were riskclassified as a high risk.

In the study population $34.5 \%$ had a diagnose of labor dystocia and oxytocin infusion initiated at $\leq 5 \mathrm{~cm}$ cervix dilatation, $31.1 \%$ at $6-10 \mathrm{~cm}$ of cervical dilatation, and $34.3 \%$ at fully dilated cervix. The three groups were similar in terms of maternal characteristics, apart from a statistically significant difference in height, BMI in early pregnancy and risk classification on admission to the labor ward (Table 1). No women had pre-pregnancy hypertension, renal disease, preeclampsia, diabetes mellitus or heart disease.

TABLE 1 Maternal and obstetric characteristics of the study population. 


\begin{tabular}{|c|c|c|c|c|}
\hline $\begin{array}{l}\text { Cervix dilatation at diagnose of dystocia and start of } \\
\text { oxytocin infusion }\end{array}$ & $\begin{array}{l}\leq 5 \mathrm{~cm} \\
\mathrm{n}=203\end{array}$ & $\begin{array}{l}6-10 \\
\mathrm{~cm} \\
\mathrm{n}=183\end{array}$ & $\begin{array}{l}\text { Fully } \\
\text { dilated } \\
n=202\end{array}$ & $\begin{array}{l}\mathrm{p}- \\
\text { value }\end{array}$ \\
\hline Age (years) mean [SD] & $\begin{array}{l}28.5 \\
{[4.8]}\end{array}$ & $\begin{array}{l}28.9 \\
{[4.3]}\end{array}$ & $29.4[4.3]$ & 0.177 \\
\hline Smoking during pregnancy $\mathrm{n}(\%)$ & $5(3)$ & $5(3)$ & $1(0.5)$ & 0.202 \\
\hline Height (cm) mean [SD] & $\begin{array}{l}164.8 \\
{[6.0]}\end{array}$ & $\begin{array}{l}166.2 \\
{[6.0]}\end{array}$ & $\begin{array}{l}167.6 \\
{[6.1]}\end{array}$ & $<0.001$ \\
\hline $\operatorname{BMI}\left(\mathrm{kg} / \mathrm{m}^{2}\right)$ mean [SD] & $\begin{array}{l}25.7 \\
{[5.1]}\end{array}$ & $\begin{array}{l}25.8 \\
{[5.2]}\end{array}$ & $24.5[4.1]$ & 0.013 \\
\hline Asthma/lung disease $\mathrm{n}(\%)$ & $21(11)$ & $8(4)$ & $18(9)$ & 0.075 \\
\hline $\begin{array}{l}\text { Gestational age in days } \\
\text { mean [SD] }\end{array}$ & $\begin{array}{l}283 \\
{[7.1]}\end{array}$ & $\begin{array}{l}282 \\
{[7.4]}\end{array}$ & $281[7.0]$ & 0.14 \\
\hline \multicolumn{5}{|l|}{ Risk assessment at admission $\mathrm{n}(\%)$} \\
\hline Low risk & $59(29)$ & $78(43)$ & $105(52)$ & $<0.001$ \\
\hline $\begin{array}{l}\text { Medium risk } \\
\text { High risk }\end{array}$ & $\begin{array}{l}144 \\
(71) \\
0\end{array}$ & $\begin{array}{l}105 \\
(57) \\
0\end{array}$ & $\begin{array}{l}97(48) \\
0\end{array}$ & \\
\hline Active phase of first stage of labor in hours, mean [SD] & $\begin{array}{l}12.1 \\
{[7.9]}\end{array}$ & $\begin{array}{l}12.0 \\
{[7.2]}\end{array}$ & $7.67[4.6]$ & $<0.001$ \\
\hline Second stage of labor in hours, mean [SD] & $\begin{array}{l}0.72 \\
{[6.0]}\end{array}$ & $\begin{array}{l}1.46 \\
{[5.9]}\end{array}$ & $2.73[3.8]$ & 0.001 \\
\hline
\end{tabular}

Data is presented as mean and [standard deviation] for continuous variables and number and (percent) for categorical variables. Percent was calculated within dilatation groups. $p<0.05$ was considered statistically significant.

$\mathrm{BMI}=$ Body mass index

The primary outcome, mode of birth in relation to cervical dilatation at diagnose of dystocia and initiation of oxytocin infusion, is presented in Tables 2 and 3.

TABLE 2 Outcomes according to cervix dilatation groups at diagnose of dystocia and start of oxytocin infusion. 


\begin{tabular}{|c|c|c|c|c|}
\hline $\begin{array}{l}\text { Cervix dilatation at diagnose of dystocia and start } \\
\text { of oxytocin infusion }\end{array}$ & $\begin{array}{l}\leq 5 \mathrm{~cm} \\
\mathrm{n}=203\end{array}$ & $\begin{array}{l}6-10 \\
\mathrm{~cm} \\
\mathrm{n}=183\end{array}$ & $\begin{array}{l}\text { Fully dilated } \\
\mathrm{n}=202\end{array}$ & $\begin{array}{l}\mathrm{p}- \\
\text { value }\end{array}$ \\
\hline Spontaneous vaginal birth $\mathrm{n}(\%)$ & $\begin{array}{l}149 \\
(73.4)\end{array}$ & $\begin{array}{l}144 \\
(78.7)\end{array}$ & $179(88.6)$ & $<0.001$ \\
\hline Instrumental vaginal birth $\mathrm{n}(\%)$ & $\begin{array}{l}29 \\
(14.3)\end{array}$ & $\begin{array}{l}27 \\
(14.8)\end{array}$ & $23(11.4)$ & 0.569 \\
\hline Cesarean section n (\%) & $\begin{array}{l}25 \\
(12.3)\end{array}$ & $\begin{array}{l}12 \\
(6.6)\end{array}$ & $0(0.0)$ & $<0.001$ \\
\hline Need of epidural anesthesia $n(\%)$ & $\begin{array}{l}178 \\
(88)\end{array}$ & $\begin{array}{l}162 \\
(89)\end{array}$ & $142(70)$ & $<0.001$ \\
\hline OASI grade III+IV n (\%) & $13(6)$ & $10(5)$ & $11(5)$ & 0.89 \\
\hline $\mathrm{PPH}(>1000 \mathrm{~mL}) \mathrm{n}(\%)$ & $14(7)$ & $10(6)$ & $14(7)$ & 0.80 \\
\hline Apgar $<7$ at $5 \min n(\%)$ & $8(4)$ & $4(2)$ & $1(0.5)$ & 0.06 \\
\hline $\begin{array}{l}\text { Umbilical cord arterial } \mathrm{pH}<7.10 \\
\mathrm{n}(\%)\end{array}$ & $\begin{array}{l}19 \\
(12.2)\end{array}$ & $\begin{array}{l}14 \\
(10.3)\end{array}$ & $15(9.7)$ & 0.76 \\
\hline $\begin{array}{l}\text { Negative birth experience } \\
\text { (VAS 1-4) n (\%) }\end{array}$ & $\begin{array}{l}53 \\
(28.5)\end{array}$ & $\begin{array}{l}33 \\
(19.0)\end{array}$ & $34(18.0)$ & 0.03 \\
\hline
\end{tabular}

Data is presented as mean and standard deviation for continuous variables and number and percent for categorical variables. Percent was calculated within dilatation groups. $p<0.05$ was considered statistically significant.

OASI = Obstetric Anal Sphincter injury, PPH= Postpartum hemorrhage, VAS = Visual Analog Scale

The overall CS rate in the study population was $6.3 \%, 13.4 \%$ had an instrumental vaginal birth and $80.4 \%$ a spontaneous vaginal birth. The CS rate differed significantly between the women with a diagnose of dystocia and start of oxytocin infusion at cervical dilatation $\leq 5 \mathrm{~cm}(12.3 \%)$ compared to the women with a cervical dilatation of $6-10 \mathrm{~cm}(6.6 \%)$ and the fully dilated group (none) $(\mathrm{p}<.001)$ (Table 2). Concurrently, there was a significant difference between the groups in women having a spontaneous vaginal birth with $73.4 \%$ in the $\leq 5 \mathrm{~cm}$ group, $78.7 \%$ in the $6-10 \mathrm{~cm}$ group and $88.6 \%$ in the fully dilated group. All instrumental vaginal births $(n=77)$ were vacuum extractions. The occurrence of instrumental birth did not differ significantly between the groups $(14.3 \%, 14.8 \%$ and $11.4 \%$ ) (Table 2$)$. The corresponding outcome rates in the three dilatation groups for low-risk women $(\mathrm{N}=242)$ were as follows, spontaneous vaginal birth $78.0 \%, 83.3 \%, 88.6 \%$, and CS $6.8 \%, 2.6 \%, 0 \%$.

Women diagnosed with dystocia and initiated oxytocin infusion when fully dilated had a decreased risk of operative birth (CS or instrumental vaginal birth) compared with women in the 6-10 cm cervical dilatation group (aOR $0.4895 \% \mathrm{Cl}$ 0.27-0.85) (Table 3). The secondary outcomes in relation to cervical 
dilatation at diagnose of dystocia and initiation of oxytocin infusion are shown in Tables 2 and 3 . The use of epidural anesthesia and negative birth experience (VAS 1-4) differed significantly between the three cervical dilatation groups (Table 2). Women with a diagnose of dystocia and start of oxytocin infusion at $\leq 5 \mathrm{~cm}$ of dilatation, had an increased risk of reporting a negative birth experience (VAS 1-4), compared to women in the 6-10 cm group (aOR 1.76 95\% Cl 1.05-2.95) (Table 3). Women in the fully dilated group had a decreased risk for having epidural anesthesia compared with women in the 6-10 cm group (aOR 0.28 95\% Cl 0.16-0.50) (Table 3).

TABLE 3 Crude and adjusted odds ratios for obstetric and neonatal outcomes

\begin{tabular}{|c|c|c|}
\hline Primary outcome & Crude OR $(95 \% \mathrm{Cl})$ & Adjusted OR* $(95 \% \mathrm{Cl})$ \\
\hline \multicolumn{3}{|l|}{ Operative birth } \\
\hline$\leq 5 \mathrm{~cm}$ & $1.34(0.84-2.14)$ & $1.28(0.78-2.08)$ \\
\hline Fully dilated & $0.47(0.27-0.83)$ & $0.48(0.27-0.85)$ \\
\hline \multicolumn{3}{|c|}{ Secondary outcome } \\
\hline \multicolumn{3}{|l|}{ Need of epidural } \\
\hline$\leq 5 \mathrm{~cm}$ & $0.92(0.5-1.71)$ & $0.91(0.48-1.73)$ \\
\hline Fully dilated & $0.31(0.18-0.53)$ & $0.28(0.16-0.50)$ \\
\hline \multicolumn{3}{|l|}{ OASI grade III+IV } \\
\hline$\leq 5 \mathrm{~cm}$ & $1.18(0.51-2.77)$ & $1.18(0.50-2.81)$ \\
\hline Fully dilated & $0.10(0.41-2.40)$ & $1.07(0.44-2.60)$ \\
\hline \multicolumn{3}{|l|}{ PPH (>1000 ml) } \\
\hline$\leq 5 \mathrm{~cm}$ & $1.28(0.56-2.96)$ & $1.39(0.60-3.28)$ \\
\hline Fully dilated & $1.29(0.56-2.98)$ & $1.36(0.58-3.17)$ \\
\hline \multicolumn{3}{|c|}{ Apgar score $<7$ at $5 \mathrm{~min}$} \\
\hline$\leq 5 \mathrm{~cm}$ & $1.86(0.55-6.27)$ & $1.57(0.45-5.46)$ \\
\hline Fully dilated & $0.23(0.03-2.01)$ & $0.22(0.02-2.00)$ \\
\hline \multicolumn{3}{|c|}{ Negative birth experience VAS 1-4 } \\
\hline$\leq 5 \mathrm{~cm}$ & $1.70(1.04-2.79)$ & $1.76(1.05-2.95)$ \\
\hline Fully dilated & $0.94(0.55-1.60)$ & $1.01(0.58-1.75)$ \\
\hline
\end{tabular}

Cervical dilatation at diagnose of dystocia and start of oxytocin augmentation and the risk of operative birth (instrumental vaginal birth or cesarean section), adverse obstetric and neonatal outcomes and risk 
of negative birthing experience. Cervical dilatation of $6-10 \mathrm{~cm}$ at diagnose of dystocia and start of oxytocin augmentation was set as reference.

$\mathrm{OR}=$ Odds Ratio, $\mathrm{OASI}$ = obstetric anal sphincter injury, VAS = Visual Analog Scale, $\mathrm{PPH}=$ postpartum hemorrhage

*Adjusted for maternal age at birth, BMI in early pregnancy and risk assessment at admission to the labor ward.

\section{Discussion}

This cohort study, including nulliparous women with singleton, term pregnancies, spontaneous onset of labor and vertex presentations (e.g. the TGCS group 1), showed an association between cervical dilatation at diagnose of labor dystocia and start of oxytocin infusion and mode of birth. There were significant differences between the three cervical dilatation groups ( $\leq 5 \mathrm{~cm}, 6-10 \mathrm{~cm}$, fully dilated) in rates of spontaneous vaginal births and CS rates, but no difference in rates of instrumental birth. The risk of operative birth (cesarean and vacuum) was significantly lower in the fully dilated group compared with the 6-10 cm group, but no increased risk could be shown in the $\leq 5 \mathrm{~cm}$ group in comparison to the 6-10 cm group.

Further, women with a diagnose of dystocia and start of oxytocin infusion at $\leq 5 \mathrm{~cm}$ had an increased risk of a negative birth experience.

These results are in line with a study by Häggsgård et al. who compared mode of birth in Robson group 1 according to the degree of cervical dilation when initiating labor augmentation with oxytocin. They found, in 464 women, that the more dilated the cervix was when initiating oxytocin augmentation, the higher the likelihood of a vaginal birth, and concluded that women who had oxytocin infusion initiated at $\leq 4 \mathrm{~cm}$ cervical dilatation had the highest risk of CS (13.6\%) (17).

In the present study population $34.5 \%$ were diagnosed with dystocia and received oxytocin infusion when the cervix dilatation was $\leq 5 \mathrm{~cm}$ and were thus in the latent phase of labor according to the ACOG definition (6) but not according to the Swedish criteria of active labor (10). In this group the CS rate was twice as high as in in the $6-10 \mathrm{~cm}$ group. Therefore, it is of interest to look at studies comparing mode of delivery outcome according to cervical dilatation degree when active labor starts. In a French study the definition of active labor was changed from 4 to 6 centimeters dilatation. This intervention lowered both the CS rates and the number of newborns having Apgar scores $<7$ at 1 minute (18). On the other hand, when the Norwegian LaPS trial cluster-randomized women in the TGCS group 1 to active labor, defined as either 4 or $6 \mathrm{~cm}$ of cervical dilatation, they found no difference in mode of delivery. However, the total CS rate in both groups decreased during the trial, from $9-10 \%$ to $6 \%$ (19). More studies are needed to evaluate both mode of delivery and neonatal outcomes in women with and without interventions due to labor dystocia at low cervical dilatation degrees. Another question is whether the rate of women being converted to induction of labor, due to prolonged latent phase, is increased if no intervention are done at low cervical dilatation degrees? 
The reason for the highest rate of CS in women being diagnosed with dystocia and treated with oxytocin at $\leq 5 \mathrm{~cm}$ could only be speculated upon. One explanation could be that the transformation of the cervical collagen structure (softening and more prone to dilatation) that occurs when the laboring woman reaches active labor might not yet have occurred (20) and the added force of stronger and longer contractions due to oxytocin augmentation might not help dilate the cervix and thereby no progress of labor.

The current study found that almost one-third of the women that were diagnosed with labor dystocia and had oxytocin augmentation initiated at $\leq 5 \mathrm{~cm}$ of dilatation had a negative birthing experience measured by VAS, compared to one out of five women with start of augmentation at 6-10 cm cervical dilatation.

Negative birthing experience has in earlier studies been associated with a long duration of labor and with CS $(1,21,22)$, oxytocin augmentation during the first stage of labor $(21,23)$, instrumental birth and PPH (21). Satisfaction with childbirth experience is a measure of quality, and should be a significant end-point according to the WHO, alongside the outcome of healthy mother and healthy baby. WHO further states that the increased medicalization of normal childbirth deprives women of their own birthing capabilities and contributes to a higher risk of a negative childbirth experience (8). The individual parts of the cascade of interventions in women with diagnosed labor dystocia has not been evaluated in relation the women's birth experience in the current study. Factors of importance could be that the use of oxytocin infusion not only restricts women's mobility during labor and birth due to the increased need of continuous CTG, but also increase the risk of more discomfort, pain (24) and need of epidural analgesia.

\section{Strengths and limitations}

This study has certain strengths and limitations. One strength is the large study population of term nulliparas with spontaneous onset of labor and oxytocin augmentation initiated during labor, enabling evaluation of outcomes in three cervical dilatation groups with cut-offs customized to the latest definitions on start of active labor. The detailed prospectively collected data on baseline evaluation of maternal comorbidity and socioeconomic factors, enabled adjustment for possible confounding factors. Another strength is the availability of manually extracted risk assessments, which made it possible to adjust outcomes for the woman's individual risk on admission to the labor ward.

The retrospective design of a study is always a limitation as the researcher has no control over the data entered into the medical files. Also, additional data that would have been valuable in the analysis (e.g. cervix dilatation on admission) was not available. Another drawback arose when it became apparent that no CS were performed in the group which had oxytocin initiated when cervix was fully dilated. A composite outcome of CS and instrumental birth was therefore created and named "operative birth", enabling the data to be further analyzed using binary logistic regression. The context in which this study was performed has a long tradition of high use of oxytocin but also high frequencies of spontaneous vaginal births, a fact that might reduce the generalizability to other populations where CS are more common. 


\section{Conclusion}

This study on nulliparous women with spontaneous onset of labor and labor dystocia, performed in a low CS setting, showed a significant difference in CS rates among the three cervical dilatation groups, with the highest rate of CS when labor dystocia was diagnosed and oxytocin was initiated before $\leq 5 \mathrm{~cm}$ of cervical dilatation. When analyzing the composite outcome of operative birth, with the $6-10 \mathrm{~cm}$ of dilatation group as a reference and adjusting for confounders, a significant lower risk of operative birth was found in the fully dilated group. The higher risk for a negative birth satisfaction among the women in the $\leq 5 \mathrm{~cm}$ of cervical dilatation group, calls for caution when considering augmenting labor at $\leq 5 \mathrm{~cm}$ of cervical dilatation. The results from the present study might support the shift toward a definition of active labor at a higher cervical dilatation degree, minimizing interventions in early stages of labor, and thus potentially increasing both the number of spontaneous vaginal births and women's satisfaction with childbirth. This should be thoroughly evaluated beforehand though, since modern labor care, at present, is not organized to handle a large number of women laboring for longer periods of time inside the hospital.

\section{Abbreviations}

ACOG American College of Obstetricians and Gynecologists

ANOVA Analysis of Variance

aORs adjusted odds ratios

BMI Body Mass Index $\left(\mathrm{kg} / \mathrm{m}^{2}\right)$.

Cls Confidence intervals

CS Cesarean section

CTG Cardiotocography

OASI Obstetric anal sphincter injury

ORs Odds ratios

$\mathrm{PPH}$ Postpartum hemorrhage

TGCS Ten Group Classification System

WHO World Health Organization

\section{Declarations}

Ethics approval and consent to participate 
The Regional Ethical Review Board in Linköping, Sweden approved the study and the extraction of data from patient medical records on June 14 ${ }^{\text {th }}, 2017$ (Dnr. 2017-277-31) and on February 19 ${ }^{\text {th }}, 2019$ (Dnr. 2019-01059). Upon collection, all data was de-identified. According to statement from the Regional Ethical Review Board, informed consent was not needed as no individuals were contacted. All methods were carried out in accordance with relevant guidelines and regulations.

\section{Consent for publication}

Not applicable.

\section{Availability of data and materials}

The datasets generated and/or analyzed during the current study are not publicly available due to restrictions in Swedish law (Offentlighets- och sekretesslag (SFS 2009:400)/Public Access to Information and Secrecy Act (SFS 2009:400)) but are available from the corresponding author on reasonable request.

\section{Competing interests}

The authors declare that they have no competing interests.

\section{Funding}

This work was supported by the ALF grants, Region Östergötland (grant no. RÖ-938175). The funder was not involved in the development of the study from the idea to the project plan, the analysis of data or writing the manuscript.

\section{Authors' contributions}

$\mathrm{CB}$ and $\mathrm{MB}$ designed the study. $\mathrm{CB}$ extracted all data from medical records. $\mathrm{CB}$ did the statistical analyses. $\mathrm{CB}, \mathrm{HG}, \mathrm{SC}$, and $\mathrm{MB}$ contributed to the analysis and interpretation of data for the work. $\mathrm{CB}$ and MB wrote the manuscript. SC and HG critically revised the manuscript. All authors gave final approval and agreed to be accountable for all aspects of work ensuring integrity and accuracy.

\section{Acknowledgements}

The authors would like to thank Lars Valter, Forum Östergötland, Linköping University and Region Östergötland, Linköping, Sweden for assistance with statistics.

\section{References}

1. Selin L, Wallin G, Berg M. Dystocia in labour-risk factors, management and outcome: a retrospective observational study in a Swedish setting. Acta Obstet Gynecol Scand. 2008;87(2):216-21.

2. Cheng YW, Shaffer BL, Bryant AS, Caughey AB. Length of the first stage of labor and associated perinatal outcomes in nulliparous women. Obstet Gynecol. 2010;116(5):1127-35. 
3. Nyfløt LT, Stray-Pedersen B, Forsén L, Vangen S. Duration of labor and the risk of severe postpartum hemorrhage: a case-control study. PloS one. 2017;12(4):e0175306.

4. Selin L, Almström E, Wallin G, Berg M. Use and abuse of oxytocin for augmentation of labor. Acta Obstet Gynecol Scand. 2009;88(12):1352-7.

5. Socialstyrelsen. Indikation för värkstimulering med oxytocin under aktiv förlossning (Indication for augmentation with oxytocin during active labor). 2011 2011 08/01. Report No.: 2011:18.

6. Caughey AB, Cahill AG, Guise J-M, Rouse DJ, Obstetricians ACo, Gynecologists. Safe prevention of the primary cesarean delivery. Am J Obstet Gynecol. 2014;210(3):179-93.

7. World Health Organization. WHO labour care guide: user's manual. Geneva; 2020.

8. World Health Organization. WHO recommendations: intrapartum care for a positive childbirth experience. Geneva; 2018.

9. Friedman EA. The graphic analysis of labor. Am J Obstet Gynecol. 1954;68(6):1568-75.

10. Perinatal ARG och svenska Barnmorskeförbundet. Definition av etablerat förlossningsarbete (Definition of active labor). 2015.

11. World Health Organization. Robson Classification: Implementation Manual. Geneva; 2017.

12. Robson M. The Ten Group Classification System (TGCS)-a common starting point for more detailed analysis. BJOG. 2015;122(5):701.

13. Boerma T, Ronsmans C, Melesse DY, Barros AJ, Barros FC, Juan L, et al. Global epidemiology of use of and disparities in caesarean sections. Lancet. 2018;392(10155):1341-8.

14. Blomberg M. Avoiding the first cesarean section-results of structured organizational and cultural changes. Acta Obstet Gynecol Scand. 2016;95(5):580-6.

15. Caughey $A B$, editor Is Zhang the new Friedman: How should we evaluate the first stage of labor? Semin Perinatol; 2020: Elsevier.

16. Graviditetsregistret (The Swedish Pregnancy Registry) [Internet]. 2020 [cited 16 Dec 2020]. Available from: https://www.medscinet.com/gr/default.aspx.

17. Häggsgård C, Persson EK. Management of oxytocin for labour augmentation in relation to mode of birth in Robson group 1. Midwifery. 2020;90:102822.

18. Thuillier C, Roy S, Peyronnet V, Quibel T, Nlandu A, Rozenberg P. Impact of recommended changes in labor management for prevention of the primary cesarean delivery. Am J Obstet Gynecol. 2018;218(3):341. e1-e9.

19. Bernitz S, Dalbye R, Zhang J, Eggebø TM, Frøslie KF, Olsen IC, et al. The frequency of intrapartum caesarean section use with the WHO partograph versus Zhang's guideline in the Labour Progression Study (LaPS): a multicentre, cluster-randomised controlled trial. Lancet. 2019;393(10169):340-8.

20. Uldbjerg N, Ekman G, Malmström A, Olsson K, Ulmsten U. Ripening of the human uterine cervix related to changes in collagen, glycosaminoglycans, and collagenolytic activity. Am J Obstet Gynecol. 1983;147(6):662-6. 
21. Falk M, Nelson M, Blomberg $M$. The impact of obstetric interventions and complications on women's satisfaction with childbirth a population based cohort study including 16,000 women. BMC Pregnancy and Childbirth. 2019;19(1):494.

22. Nystedt A, Hildingsson I. Diverse definitions of prolonged labour and its consequences with sometimes subsequent inappropriate treatment. BMC Pregnancy Childbirth. 2014;14(1):233.

23. Johansson C, Finnbogadóttir H. First-time mothers' satisfaction with their birth experience-a crosssectional study. Midwifery. 2019;79:102540.

24. Wei S-Q, Luo Z-C, Xu H, Fraser WD. The effect of early oxytocin augmentation in labor: a metaanalysis. Obstet Gynecol. 2009;114(3):641-9.

\section{Figures}




\section{Women eligible for the study:}

Nulliparous women at term with a singleton pregnancy, vertex presentation and spontaneous onset of labor $(n=$ 1,021) giving birth between March 28 , Women excluded from the study: 2018 and November 1, 2018.

Women not receiving oxytocin augmentation. $n=340$.

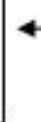

Women not risk-assessed on admission to the labor ward. $n=$ 70 .

Women diagnosed both as spontaneous onset of labor and induction of labor. $n=23$

\section{Women included in the study:}

Nulliparous women at term with a singleton pregnancy, vertex presentation, and spontaneous onset of labour, were risk-assessed on admission to the labor ward and a diagnose of dystocia requiring oxytocin infusion during labor. $n=588$.

\begin{tabular}{|l|l|l|}
\hline $\begin{array}{l}\text { Dystocia } \\
\text { diagnosed and } \\
\text { oxytocin infusion } \\
\text { initiated at } \\
\text { cervical dilatation } \\
\leq 5 \mathrm{~cm} . \mathrm{n}=203\end{array}$ & $\begin{array}{l}\text { Dystocia } \\
\text { diagnosed and } \\
\text { oxytocin infusion } \\
\text { initiated at } \\
\text { cervical dilatation } \\
6-10 \mathrm{~cm} . \mathrm{n}=183\end{array}$ \\
\hline
\end{tabular}

\section{Figure 1}

Flowchart of the study population

\section{Supplementary Files}

This is a list of supplementary files associated with this preprint. Click to download.

- APPENDIX1.docx

- Supplementaryinformation.docx 